\title{
Study of Bi-level Resist System with Conductive Bottom Layer for EB lithography
}

\author{
Keiji Watanabe, Miwa Igarashi, Ei Yano, \\ Eiichi Hoshino*, Kotaro Shirabe**, and Masafumi Nakaishi** \\ Fujitsu Laboratories Ltd. \\ 10-1 Morinosato-Wakamiya, Atsugi, 243-01, Japan \\ FUJITSU LIMITED \\ *1015 Kamikodanaka, nakahara-ku, Kawasaki, 211, Japan \\ **1500 Mizono, Tado-cho, Kuwana-gun, Mie 511-01, Japan
}

\begin{abstract}
We describe a new electron-beam (EB) bi-level resist system of threedimensional polysilphenylenesiloxane (TSPS) over a conductive bottom layer containing sulfonated polyaniline (SPAn). The top-layer TSPS, with a chloromethylphenylethyl (CMPE) functional group and a suitable molecular weight, is a highly sensitive negative resist without a chemically amplified system, and has a long film life. The conductive bottom layer, comprises SPAn and a crosslinker, and has excellent conductivity, a long shelf-life, and stability to solvents used for TSPS coating and development. We achieved a high resolution for 1-Gbit DRAM reticle fabrication with optical proximity correction (OPC) patterns, and significantly reduced the pattern distortion caused by the proximity effect and EB charging using this system without any data correction. Moreover, we found that the conductive resist system can be used to reduce the plasma etching damage of thin gate oxide when used in MOS-LSI device fabrication.
\end{abstract}

\section{Introduction}

Electron beam (EB) lithography is widely used in advanced device developments, application-specific integrated circuit (ASIC) applications, and photomask fabrication, due to its characteristics, including its sub- $0.1 \mu \mathrm{m}$ pattern resolution, pattern generating function, and distortion correcting function. Recently, high-throughput EB exposure systems have been investigated for the mass production of LSI devices[1],[2]. However, EB lithography suffers from problems such as proximity effects and EB charging. These prevent the pattern from being resolved as designed. The proximity effect can be solved by using exposure data with data crrection. However this would excessively increase the amount of exposure data. Therefore, EB lithography needs a faithful pattern resolution without using any data correction, and this is only possible using special resist materials and systems.

Surface imaging techniques with a thick resist film are useful for reducing the proximity effects [3]. However, increasing the resist thickness causes resist charging. One possibility is bi-level resist system [4] with an electro-conducutive bottom layer to prevent or reduce charging [5]. The bi-level resist method has many merits compared other surface imaging methods. It ts an easier process than the tri-level method, and has a more rectangular surface imaging pattern 
fabrication ability than the surface silylation method. Also, it is suitable for fabricating conductive bottom layers with no photosensitivity. In the previous study, we proposed a bilevel resist system with chemically amplified (CA) silicon containing resist over the conductive bottom layer consisting of the tetracyanoquinodimethane (TCNQ) complex salt, and exhibited a high resolution with charge reducing effect [6].

We describe a new bi-level resist system with a high performance siloxane resist and a conductive bottom layer containing sulfonated polyaniline (SPAn) for practical use in photomask fabrication. The photomask fabrication process needs a long film life (the stability to post coating delay) and a high charge reducing ability compared with the silicon wafer process. This new system consists of highly sensitive siloxane resist with no CA resist system for long film life. It also has a highly conductive bottom layer containing SPAn for the high charge reduction ability. In addition, we talk about a new application for the conductive resist system, to reduce plasma etching damage of the thin gate oxide in the silicon wafer process.

\section{Experiments}

\subsection{Preparation and evaluation of samples}

The top-imaging layer siloxane resists, three-dimensional polysilphenylenesiloxane (TSPS, Fig.1) with several functional groups, were synthesized[7]. The TSPS resists with valious functional groups were obtained by changing the triorganochlorosilane feed ratio in the syntheses. The TSPS with defferent molecular weights were obtained by fractionation of crude TSPS polymer with a broad molecular weight distribution. The functional group contents of the resists were evaluated from NMR (model JMN-GX-500, Jeol) and the molecular weights were determined by GPC (model HLC-8020, Tosoh) calibrated with polystyrene standard. We dissolved the TSPS resists in methylisobutylketone (MIBK), then spin-coated with a thickness of $0.2 \mu \mathrm{m}$ on the substrate, and prebaked at $90^{\circ} \mathrm{C}$ for 1 minute on a hot-plate. The EB sensitivities of the resists were evaluated by an EB exposure system (model ELS-3300LB, Elionix) with an acceleration voltage of $30 \mathrm{kV}$. The exposed resists were developed with an anisole and isopropanol (IPA) solution.
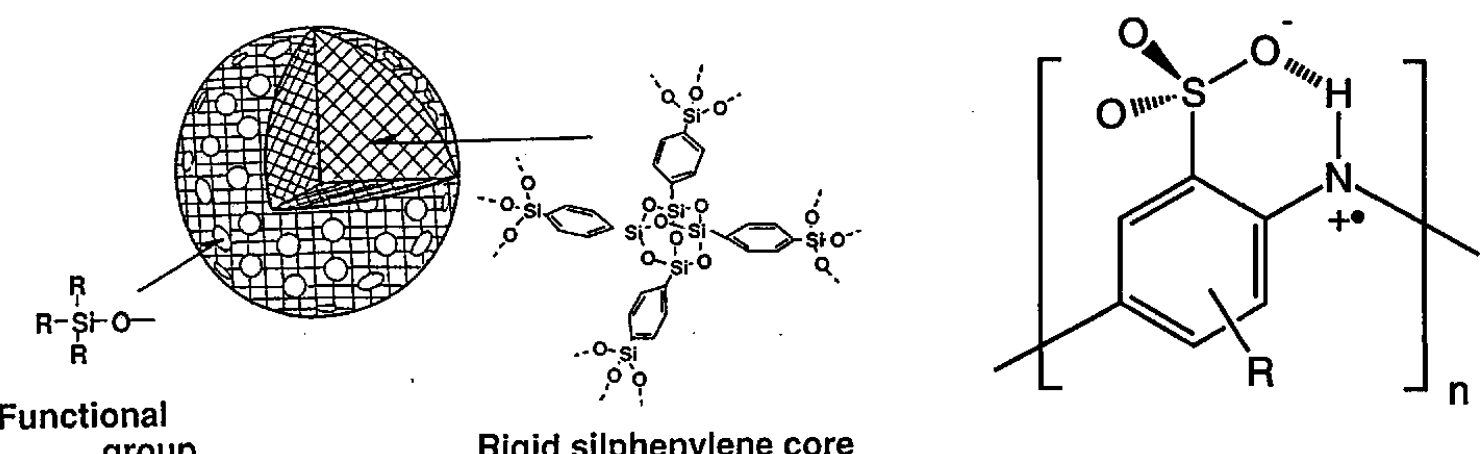

group

Rigid silphenylene core

Fig. 1. Structure model of TSPS. Fig. 2. Molecular structure of SPAn. 
The conductive bottom layer, consisting of SPAn (Fig. 2), crosslinker, and solvent were prepared by Nitto Chemical Industry Co. Ltd. We spin-coated the conductive films with a thickness of $1.5 \mu \mathrm{m}$ on the substrate, and prebaked under several conditions on the hot-plate. The sheet resistances of the conductive films were measured by an MCP tester (model MCPHT250, Mitsubishi Yuka).

\subsection{Photomask fabrication}

Figure 3 shows the process flow from the fabrication of the photomask substrate. The conductive bottom layer containing SPAn and a TSPS-3 (Table.1) resist layer were spin-coated with a $1.5 \mu \mathrm{m}$ and $0.2 \mu \mathrm{m}$-thick films on the chromium reticle substrate. After EB exposure (acceleration voltage of $20 \mathrm{kV}$, current density of $10 \mathrm{~A} / \mathrm{cm}^{2}$ ), the upper TSPS film was developed. Then the TSPS resist patterns were transferred to the conductive bottom layer by using $\mathrm{O}_{2}$-RIE (10 mTorr, $100 \mathrm{~W}, 20 \mathrm{~min}$ ). Finally, we dry-etched the chromium layer to produce chromium patterns $\left(\mathrm{CH}_{2} \mathrm{Cl}_{2}-95 \mathrm{cc} / \mathrm{min}, \mathrm{O}_{2}-55 \mathrm{cc} / \mathrm{min}, 0.24 \mathrm{Torr}, 300 \mathrm{~W}\right)$. The processed substrate was dipped in distilled water to strip the resist patterns.

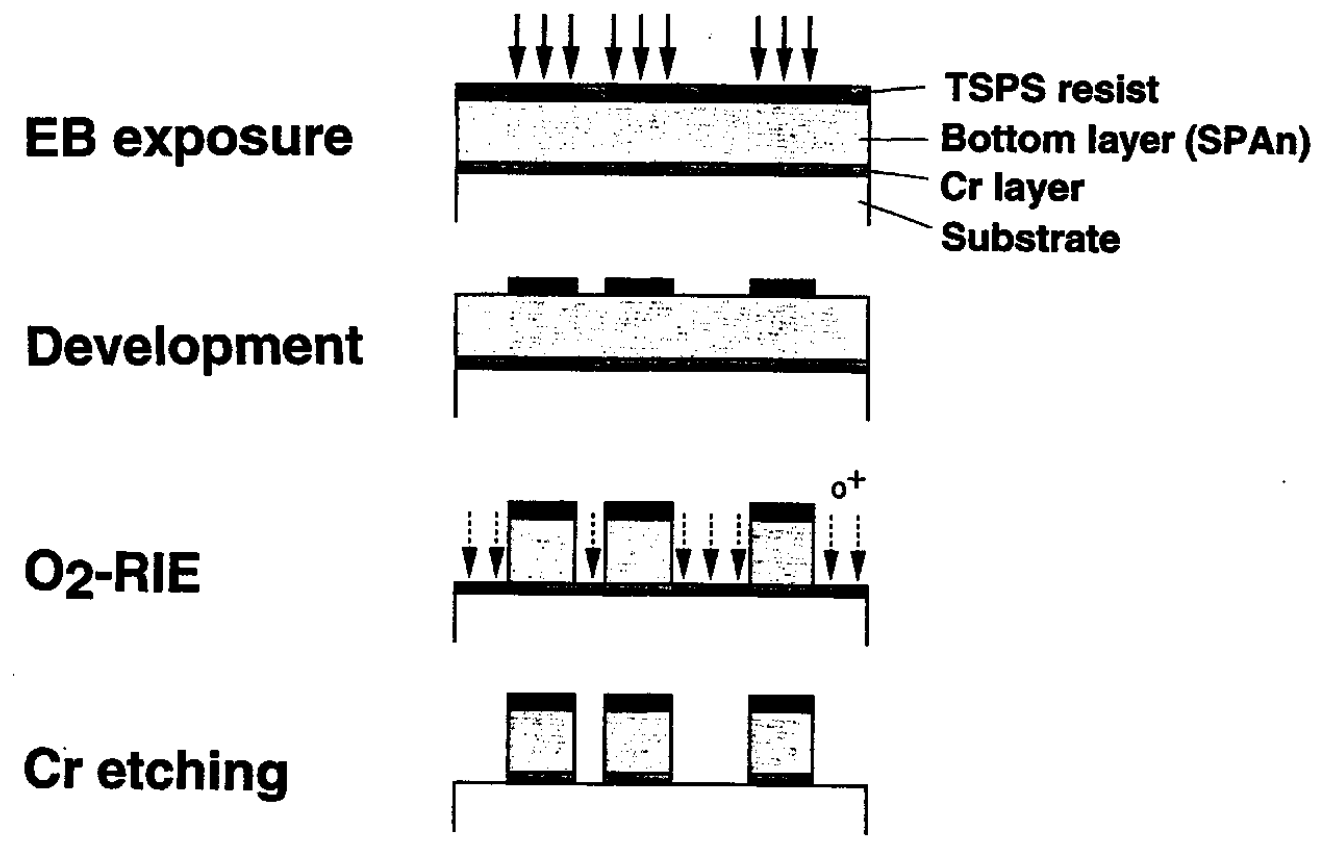

Fig. 3. Bi-level resist process for photomask fabrication.

\section{Results and Discussion}

\subsection{Highly EB-sensitive siloxane resist}

In our previous study, we reported that TSPS resist with a methyl (Me) functional group is a negative resist with conventional crosslinking mechanism, with a sub-0.1 $\mu \mathrm{m}$ resolution [7]. The TSPS molecule is structured as a rigid three-dimensional mesh consisting of a shilphenylenesiloxane core surrounded by functional groups (Fig. 1). The advantages of such a structure are high contrast, low swelling, high $\mathrm{O}_{2}$-RIE resistance, and high softening temperature. In general, these conventional resists have a longer film life time and a lower 
sensitivity than the CA resists. Therefore, we have investigated the improvement of the TSPS sensitivity selecting a suitable functional group and molecular weight.

TSPS resist containing a chloromethylphenylethyl (CMPE) group exhibits a high crosslinking efficiency when ArF excimer laser is irradiated, because of the long lifetime of the methyl radical caused by the chlorine dissociation [8]. In addition, the chloromethylphenyl functional group is known to be a highly sensitive functional group for EB negative resists [9],[10]. Therefore we expect the CMPE group to exhibit a high EB sensitivity. Table 1 shows the EB sensitivity of the TSPS with various CMPE/Me functional groups ratio and with a weight-avarage molecular weight $(M w)$ of between 15000 and 19000 , and a dispersion of between 1.6 and 1.9. As the CMPE introduction ratio increases, the TSPS sensitivity increases and the contrast decreases. About 20 to $30 \%$ of the CMPE content exhibits high sensitivity with a sub-0.1 $\mu \mathrm{m}$ resolution.

Table 1 EB sensitivity of TSPS for various CMPE/Me ratios

\begin{tabular}{|c|c|c|c|c|c|c|c|}
\hline \multirow{2}{*}{ No. } & \multirow{2}{*}{$\begin{array}{c}\text { Functional } \\
\text { group ratio } \\
\text { (CMPE/Me } \\
\mathrm{mol} / \mathrm{mol})\end{array}$} & \multicolumn{2}{|c|}{ Molecular weight } & \multicolumn{2}{|c|}{$\begin{array}{c}\text { EB sensitivity } \\
\left(\mathrm{uC} / \mathrm{cm}^{2}\right)\end{array}$} & \multirow[t]{2}{*}{$r$-value } & \multirow[t]{2}{*}{$M w \times D g^{i}$} \\
\hline & & $M w$ & $M w / M n$ & $\mathrm{Dg}^{\mathbf{i}^{*}}$ & $\mathrm{Dg} 50^{* \star}$ & & \\
\hline 1 & $0 / 100$ & 1.5E4 & 1.9 & 54 & 77 & 3.2 & 0.81 \\
\hline 2 & $8 / 92$ & $1.5 \mathrm{E} 4$ & 1.9 & 12 & 22 & 2.2 & 0.18 \\
\hline 3 & $20 / 80$ & 1.9E4 & 1.7 & 5.4 & 9.2 & 1.9 & 0.10 \\
\hline 4 & $30 / 70$ & $1.6 E 4$ & 1.6 & 4.0 & 7.8 & 1.7 & 0.064 \\
\hline
\end{tabular}

* $D g^{i}$ : Starting exposure dose of gelation

${ }^{*} \mathrm{Dg} 50$ : Exposure dose which leaves $\mathbf{5 0 \%}$ resist thickness remaining

Table 2 shows the EB sensitivity for different TSPS molecular weights. The TSPS with a high $M w$ exhibits high EB sensitivity. And the value of the $M w$ multiplied $\mathrm{D}_{\mathrm{g}}{ }^{\mathrm{i}}$ of the TSPS is decreased with the $M w$, although that of resists with conventional linear or ladder structures are constant [10],[11] Generally, the improvement in the sensitivity of the crosslinking type resist from increasing the $M w$ degrades the pattern resolution. This is because of increased swelling and pattern edge roughness caused by the effect of the large particle size. However, the degree of resolution degradation of the TSPS by the $M w$ increase is lower than that of conventional linear or ladder resists, due to its low value of the $M w$ multiplied by $\mathrm{D}_{\mathrm{g}} \mathrm{i}$. Figure 4 shows the bi-level resist pattern . $0.20 \mu \mathrm{m}$ line-and-space patterns are delineated at a dose of $3.2 \mu \mathrm{C} / \mathrm{cm}^{2}$ from using TSPS-No.7. The compatibility of sensitivity and resolution is realized by the threedimensional TSPS structure. 
Table 2 EB sensitivity of TSPS for various molecular weights

\begin{tabular}{|c|c|c|c|c|c|c|c|}
\hline \multirow[t]{2}{*}{ No. } & \multirow{2}{*}{$\begin{array}{c}\text { Functional } \\
\text { group ratio } \\
\text { (CMPE/Me } \\
\mathrm{mol} / \mathrm{mol} \text { ) }\end{array}$} & Molecul & Ir weight & \multicolumn{2}{|c|}{$\begin{array}{c}\text { EB sensitivity } \\
\left(\mathrm{uC} / \mathrm{cm}^{2}\right)\end{array}$} & \multirow[t]{2}{*}{$r$-value } & \multirow[t]{2}{*}{$M w \times D g^{i}$} \\
\hline & & $M w$ & $M w / M n$ & $\mathbf{D g}$ & $\mathrm{Dg}^{50}$ & & \\
\hline 5 & & 1.3E4 & 1.3 & 6.0 & 10.3 & 2.1 & 0.078 \\
\hline 6 & $30 / 70$ & 2.4E4 & 1.8 & 2.3 & 5.6 & 1.4 & 0.055 \\
\hline 7 & $\begin{array}{l}\text { (Crude } \\
\text { polymer) }\end{array}$ & 4.1E4 & 2.4 & 0.21 & 0.49 & 1.3 & 0.0082 \\
\hline 8 & & 4.9E4 & 1.9 & 0.15 & 0.35 & 1.4 & 0.0074 \\
\hline
\end{tabular}

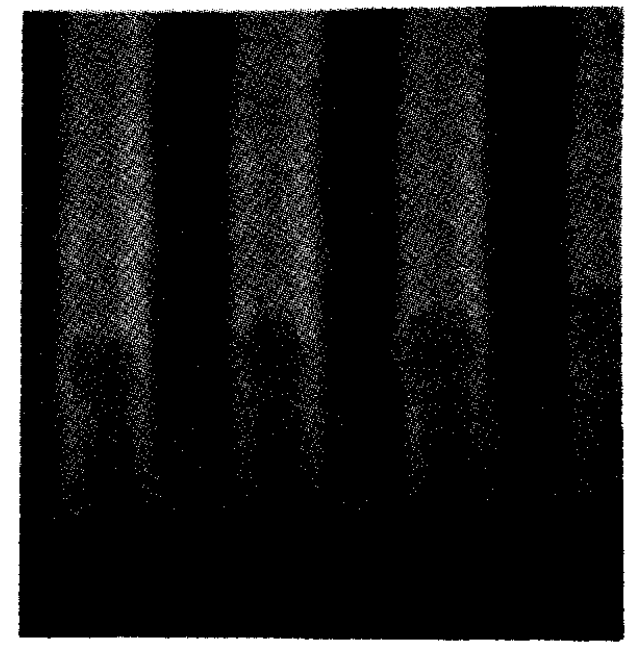

$0.200 \mu \mathrm{m} \mathrm{l} / \mathrm{s}$ patterns obtained with TSPS-7 at a dose of $3.2 \mu \mathrm{C} / \mathrm{cm}^{2}$
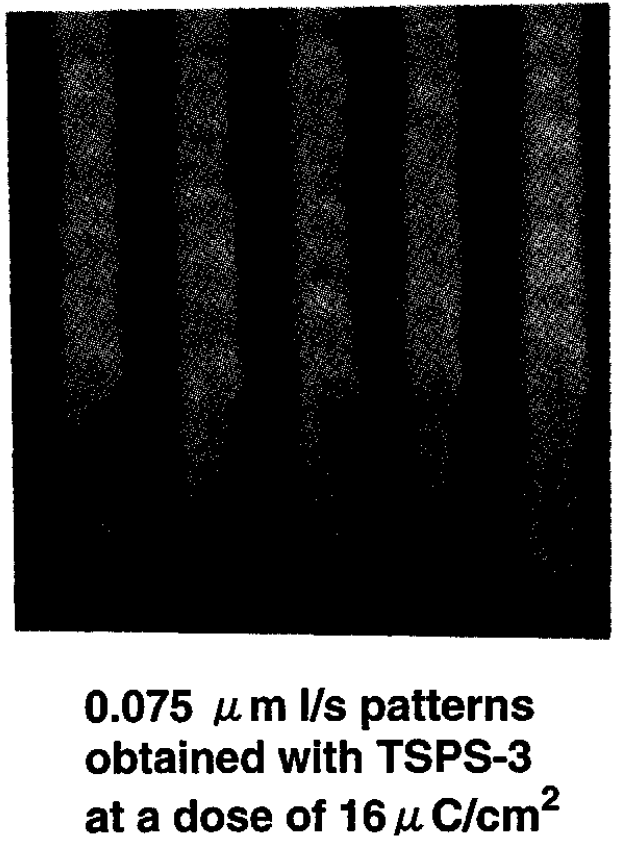

Fig. 4. Bi-level resist patterns.

\subsection{Highly conductive bottom layer}

The conductive bottom layer must meet many requirements to be suitable for fabricating fine bi-level resist patterns. Requirements include high conductivity, long shelf-life, long film-life, high solvent stability for the top-resist coating and development, and easy process for film formation. However, the bottom layer containing TCNQ in our previous study [6], had a few problems for practical use, such as a short conductivity shelf-life and a complicated film formation process. To solve the problems, we studied the conductive bottom layer by using SPAn. We investigated SPAn as a conductive top over coating of monolayer resists, and 
exhibited its high conductivity and long shelf life [12]. For solvent stability, we added a crosslinker to the SPAn. Table 3 shows the characteristics of the SPAn type conductive films compared with the TCNQ type. The SPAn bottom layer exhibits other excellent characteristics, such as high conductivity, high solvent stability, and long shelf life, with an easy film formation process.

Table 3 Comparison of characteristics of conductive bottom layers

\begin{tabular}{|c|l|l|}
\hline & TCNQ type & SPAn type \\
\hline Conductivity & $\sim 10^{7} \Omega / \square$ & $\sim 10^{5} \Omega / \square$ \\
\hline Solvent resistance & Good & Good \\
\hline Shelf life (room temp.) & $\sim 3$ week & $>3$ month \\
\hline $\begin{array}{l}\text { Film formation } \\
\text { process }\end{array}$ & $\begin{array}{l}\text { 1.Spin coat } \\
\text { 2.Bake } \\
\text { 3.Exposure } \\
\text { 4.Bake }\end{array}$ & $\begin{array}{l}\text { 1.Spin coat } \\
\text { 2.Bake }\end{array}$ \\
\hline
\end{tabular}

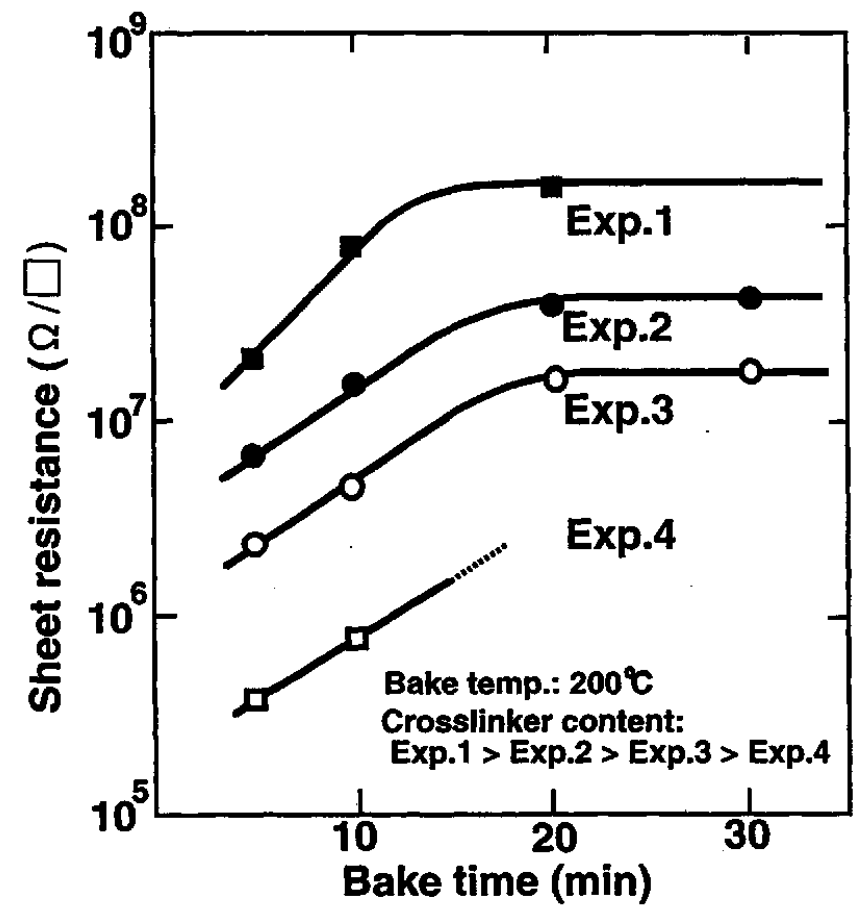

Fig. 5. Sheet resistance vs baking time of conductive bottom layer. 
Figure 5 shows the relationship between the sheet resistance with the baking time of the conductive bottom layer with several SPAn/crosslinker ratios. The sheet resistance increases with baking time and crosslinker content, and saturates at about 20 minutes. However, these keep a high level of conductivity after crosslinkage due to the high initial conductivity of SPAn. The high conductivity generation mechanism of the SPAn is responsible for the formation of the effective self-doping structure caused by the strong intermolecular interaction between the sulfonic asid group and the amino group (Fig. 2) [13]. Moreover, the interaction reduces the substrate damage caused by the sulfonic acid.

\subsection{Application for 1-Gbit DRAM reticle fabrication}

We investigated the resist system for photomask fabrication [14]. Figure 6 shows the results of CD linearity observation on the bi-level photomask process. Lines with widths from 0.5 to $5 \mu \mathrm{m}$ had a linearities within $0.1 \mu \mathrm{m}$.

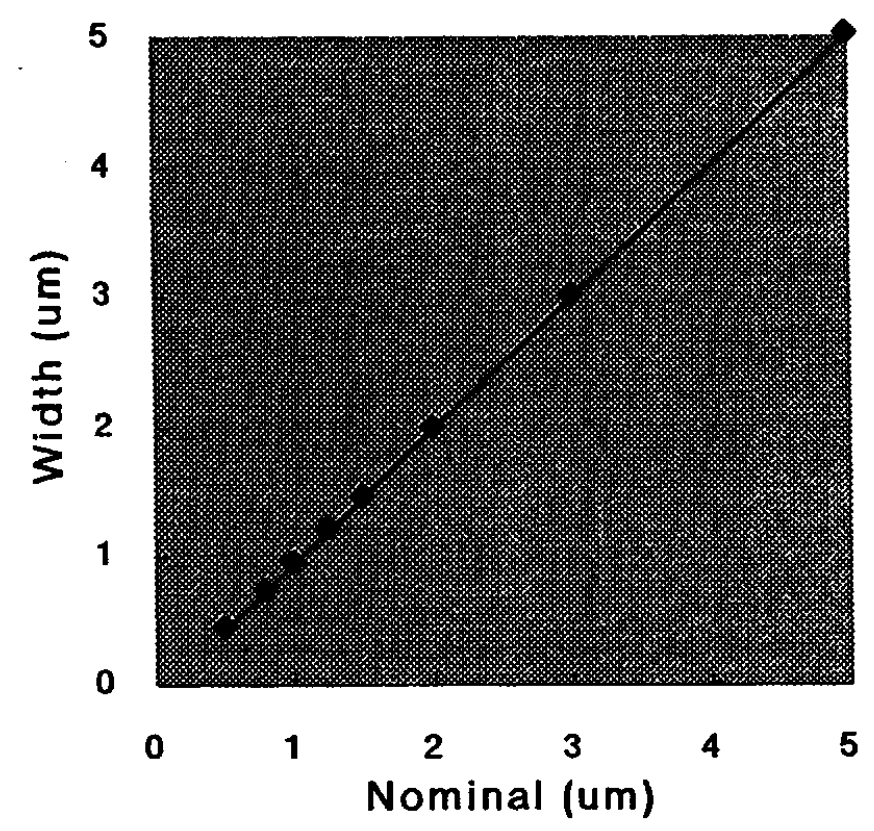

Fig. 6. CD linearity of the bi-level resist.

Figure 7 shows optical proximity correction (OPC [15]) reticle patterns for a 1-Gbit DRAM device, obtained with the conductive bi-level resist system and conventional monolayer resist. We succeeded in fabricating an OPC pattern according to the design by using the bi-level resist, due to the proximity effect reduction. We could obtain high accurate patterns, which are almost equal to nominal (Fig. 8).

Figure 9 compares the reticles of from the conventional $(0.5 \mu \mathrm{m}$-thick $)$ and bi-level $(1.7 \mu \mathrm{m}$ thick) process as in the main field placement accuracy of $15 \times 15$ squares with $1.4 \mathrm{~mm}$ side each at the center of the plate. In general, when the resist thickness is increased, placement accuracy deteriorates due to charging. However, our experiment has proved that there is no significant difference in the placement accuracy between thin conventional resist and thick bi-level resist. This is due to the high conductivity of the bottom layer containing SPAn. 


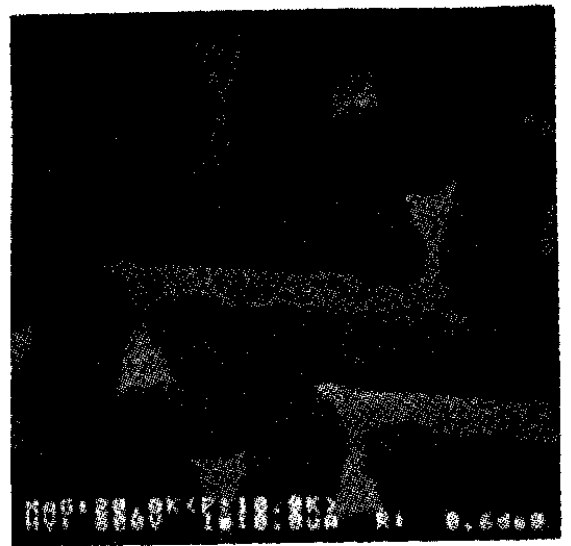

Conventional resist (proximity effect)

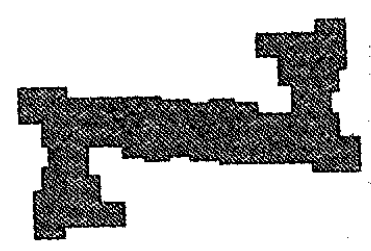

Design data

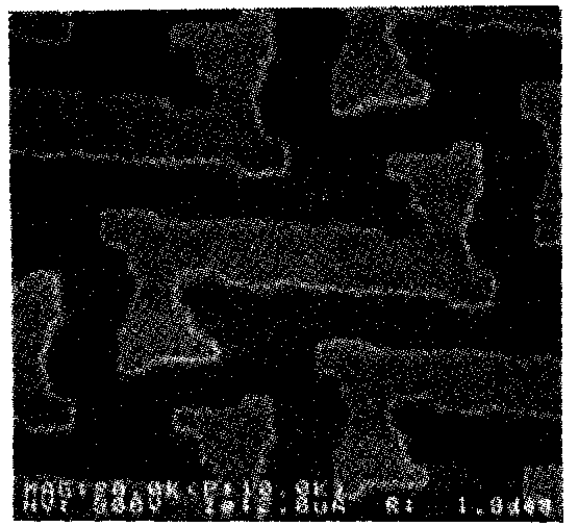

Bi-level resist

Fig. 7. OPC reticle patterns.

$0.201 \mu \mathrm{m}$

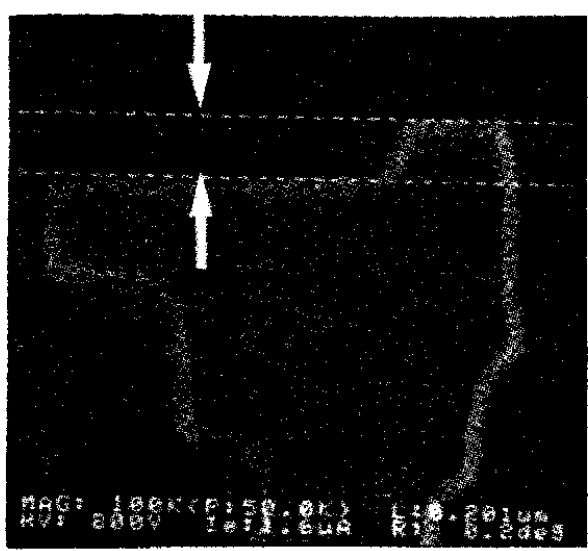

$0.155 \mu \mathrm{m}$

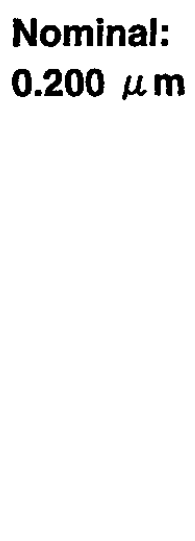

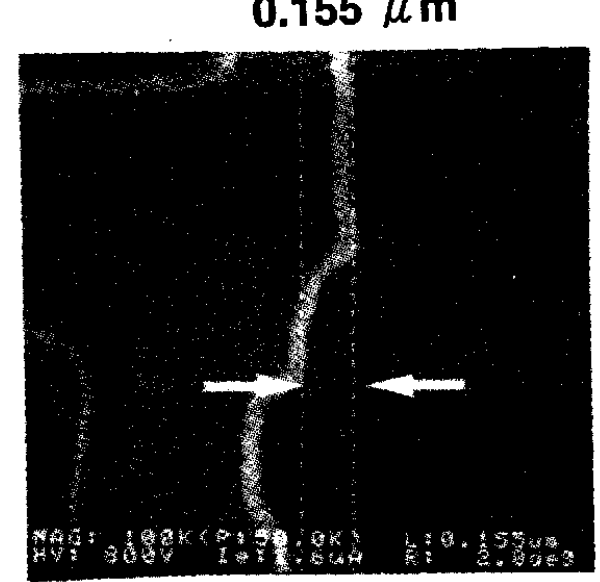

Nominal:

$0.160 \mu \mathrm{m}$

Fig. 8. OPC reticle patterns obtained with bi-level resist.
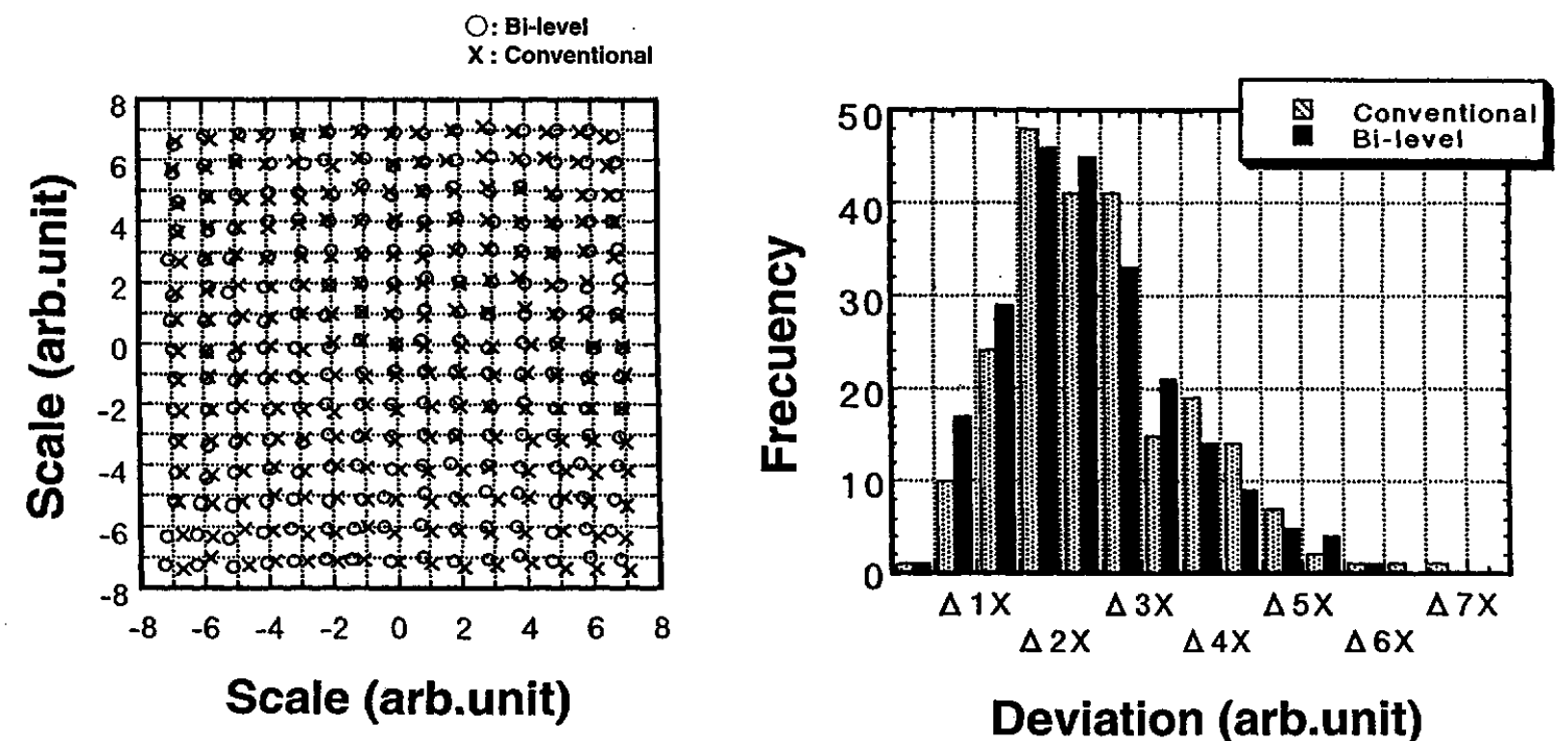

Fig. 9. Comparison of placement accuracy. 


\subsection{Application for plasma etching damage reduction}

The charge buildup during plasma etching causes gate oxide leakage or breakdown due to plasma nonuniformities. Figure 10 shows the mechanism of thin gate oxide damage in plasma etching [16]. In plasma etching, the negative charge buildup occures on the surface of resist patterns, and local ion/electron current imbalance are occurred (electron shading effect). Near the end point of etching, the positive charge buildup to the gate, and the thin-oxide damage from tunneling current through a weakened oxide area. We found that the damage can be reduced by using the conductive resists [17],[18].

Figure 11 shows the MOS capacitor yields after plasma etching, with several antenna ratios (wiring area / gate area). We used resists with several conductivities. The sample structure, we used, was a MOS capacitor with an 80-nm-thick gate oxide, and we evaluated the degree of the damage after plasma etching (ECR, bias freq. $=13.56 \mathrm{MHz}$ ) of $\mathrm{Al}$ wired for the structure. The yields were obtained by measuring I(gate oxide)-V(gate) for the MOS capacitor, and we judged the breakdown by $\mathrm{V}$ (gate) $<5 \mathrm{~V}$. The thickness of the $\mathrm{Al}$ wiring was $0.7 \mu \mathrm{m}$, and the resist patterns $(0.59 \mu \mathrm{m}$ space $)$ were obtained with the tri-level resist with the SPAn bottom layer and conventional novolak monolayer resist as the reference. The aspect-ratio of the resists after etching was 3.4. High MOS capacitor yields of more than $75 \%$ were obtained by conductive resists with the hard condition of $0 \%$ yield used by conventional novolak resist. This suggests that the damage can be reduced by using a conductive resist, without using special plasma etching apparatus or techniques.

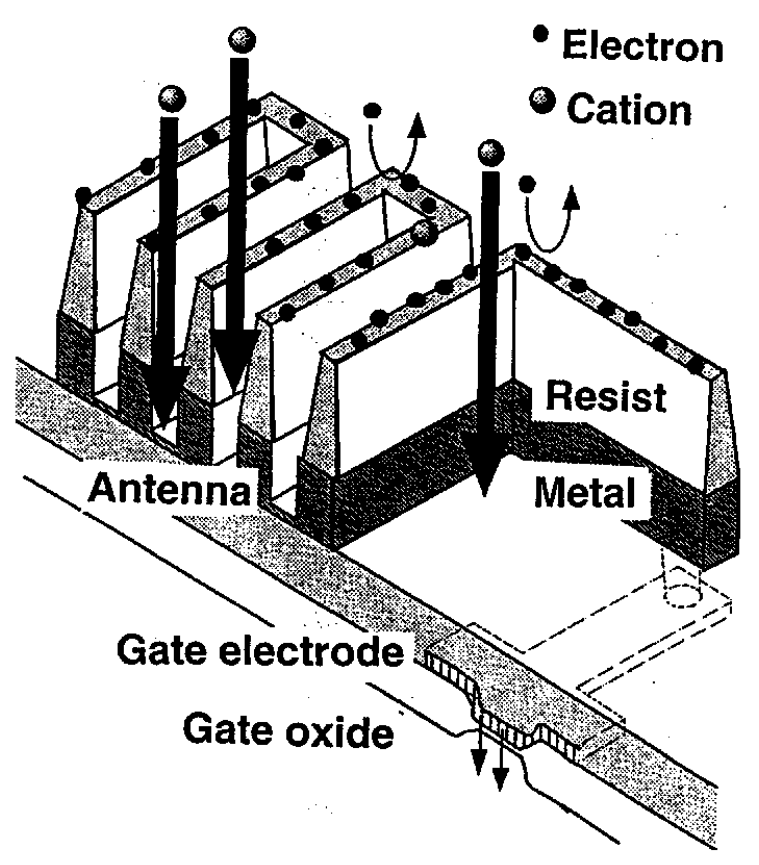

Fig. 10. Gate oxide damage mechanism.

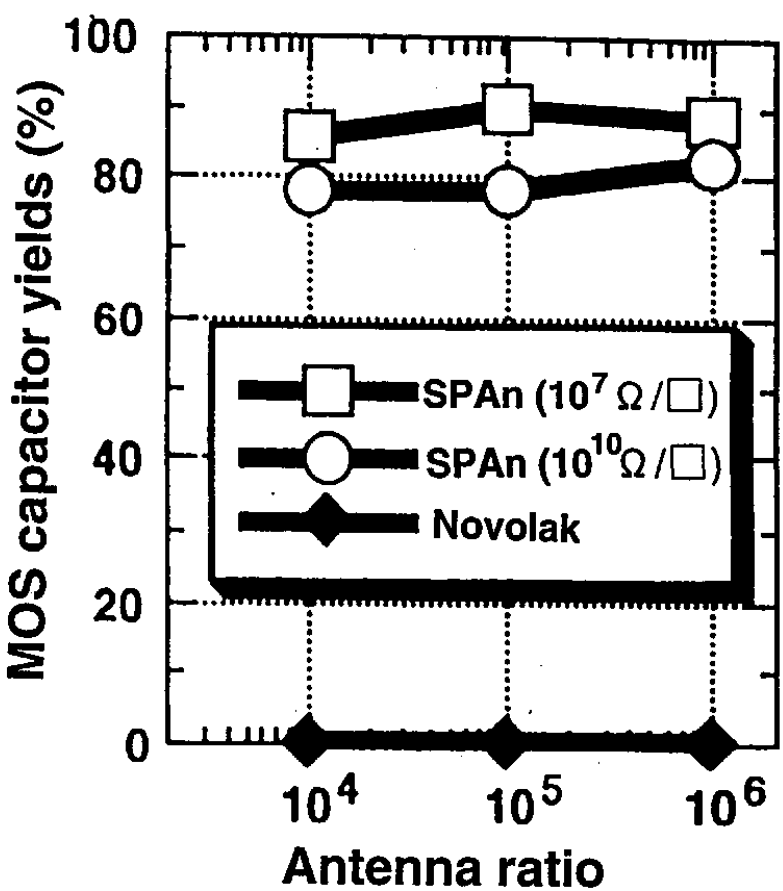

Fig. 11. MOS capacitor yields. 


\section{Conclusion}

We propose a new bi-level EB resist system using TSPS over a conductive bottom layer containing SPAn. The TSPS negative resist with a CMPE functional group and a suitable molecular weight, has a high EB sensitivity without a CA mechanism, and has a long film life time. The TSPS can resolve $0.075 \mu \mathrm{m} 1 / \mathrm{s}$ patterns at a dose of $16 \mu \mathrm{C} / \mathrm{cm}^{2}$ and $0.2 \mu \mathrm{m} 1 / \mathrm{s}$ patterns at a dose of $3.2 \mu \mathrm{C} / \mathrm{cm}^{2}$, due to the characteristics of the three-dimensional structure. The conductive bottom layer, containing a SPAn and a crosslinker, has excellent conductivity, long shelf-life, and resistance to solvents used for TSPS coating and development. The bi-level resist system has a high resolution and high aspect ratio, and significantly reduces the pattern distortion caused by the proximity effect and EB charging. With this resist system, it is possible to produce reticles with OPC patterns for 1-Gbit DRAM devices without any data correction. In addition, it can be used to reduce the plasma etching damage of the thin gate oxide when used in MOS-LSI fabrication processes.

\section{Acknowledgments}

We thank Mr. Shimizu, Ms. Yuasa, and Mr. Uzawa of Nitto Chemical Industry Co. Ltd.for assistance with conductive materials. We also wish to thank Ms. Yano and Mr. Maruyama of Fujitsu Ltd. for their help and useful advice about lithography process.

\section{References}

1) K. Sakamoto ei al., Jpn. J. Appl. Phys. 30, 6006 (1993)

2) H. Yasuda et al., Jpn. J. Appl. Phys. 32, 6012 (1993)

3) E. Hoshino et al., SEMI JAPAN, Microlithography '93, 75 (1993)

4) B. J. Lin, Solid State Technol. 26, 5, 105 (1983)

5) Y. Kawasaki et al. Extended Abstracts of JSAP 47th Autumn Meeting, 556 (1986)

6) K. Watanabe et al: J.Photopolym. Sci.Technol.,Vol. 6, No.1 pp. 57-62 (1993)

7) K. Watanabe et al: ACS Symposium Series, 537, 194 (1994)

8) M. Morita et al., J. Electrochem. Sos. 131 (1984)

9) A. Tanaka et al., REVIEW of the Electrical Communication Laboratories, Vol.33, 5 (1985)

10) K. Watanabe et al: J.Photopolym. Sci.Technol.,Vol. 8, No.1 pp. 11-20 (1995)

11) K. Watanabe et al: J.Photopolym. Sci.Technol.,Vol..1, No.1 pp. 71-78 (1988)

12) K. Yano et al., Extended Abstracts of JSAP 42th Spring Meeting, 634 (1995)

13) S. Shimizu et al., Extended Abstracts of JSAP 56th Autumn Meeting, 634 (1995)

14) K. Shirabe et al., Proc. of Photomask Japan ‘96, 2793-02 (1996)

15) O. W. Otto et al., Proc. of SPIE 2179, 278

16) K. Hashimoto et al., Jpn. J Appl. Phys., 33, 10, pp.6013-6018 (1994)

17) M. Nakaishi et al., Extended Abstracts of JSAP 56th Autumn Meeting, 513 (1995)

18) K. Watanabe et al., Extended Abstracts of JSAP 56th Autumn Meeting, 634 (1995) 